\title{
Editorial
}

\section{Epidemiologia Descritiva: qualidade das informações e pesquisa nos serviços de saúde}

$\mathrm{D}$ entre as etapas fundamentais da pesquisa epidemiológica destaca-se a descrição detalhada da ocorrência de eventos relacionados à saúde nas populações. Com a intenção de conhecer padrões gerais no comportamento de doenças e identificar subgrupos populacionais mais vulneráveis, lança-se mão de estratégias analíticas que têm como principais elementos a distribuição temporal, espacial e segundo atributos pessoais. ${ }^{1}$ A importância destas abordagens para a compreensão de fenômenos epidemiológicos, o que se convencionou denominar Epidemiologia descritiva, é salientada nos textos clássicos da disciplina, usualmente sob o título "descrição das doenças segundo pessoa-tempo-lugar" ${ }^{2,3}$ A partir da segunda metade do século XX, no contexto do surgimento e estabelecimento da assim chamada Epidemiologia Moderna, o interesse científico nestes tipos de estudo foi decrescendo paulatinamente na medida em que as pesquisas epidemiológicas foram se orientando progressivamente para a investigação das causas biológicas das doenças, através dos desenhos de estudos denominados analíticos. ${ }^{1,4}$ Ainda que abordagens descritivas e analíticas sejam freqüentemente consideradas mutuamente exclusivas, elas devem ser vistas como estratégias complementares que se dispõem em um continuum ${ }^{5,6}$ Esta falsa dicotomia entre estudos descritivos e analíticos não contribui para o desenvolvimento do campo da Epidemiologia, particularmente na sua interface com os serviços de saúde. Investigações de cunho mais descritivo configuram um arsenal metodológico fundamental para a compreensão de problemas de saúde, sendo que uma série de aspectos relacionados à concepção, desenho e análise destes estudos oferecem desafios tão ou mais complexos do que aqueles enfrentados nos demais tipos de delineamentos epidemiológicos. ${ }^{1}$ Dentre estes tantos desafios destaca-se a necessidade de se atentar para a qualidade do processo de obtenção de dados, em particular quando os sistemas de informações em saúde são utilizados como fontes de dados das pesquisas. Esta terceira edição da Epidemiologia e Serviços de Saúde no ano de 2009 traz, além de uma republicação, oito artigos originais que exemplificam a importância da Epidemiologia Descritiva e da avaliação da qualidade das informações para as pesquisas epidemiológicas.

Seis artigos utilizam abordagens descritivas para tratar de temas de grande interesse epidemiológico. Campagna e colaboradores ${ }^{7}$ utilizam dados do Sistema de Informações sobre Mortalidade (SIM) para descrever a magnitude e as variações temporais da mortalidade por doenças respiratórias relacionadas à influenza - pneumonias, influenza, bronquites e obstrução crônica das vias respiratórias - em pessoas com 60 anos de idade ou mais no Brasil no período de 1992 a 2005. Os resultados indicam taxas de mortalidade mais elevadas entre os indivíduos com 80 anos de idade ou mais, assim como entre os homens, em todas as faixas etárias; e principalmente entre os residentes nas Regiões Sul, Sudeste e Centro-Oeste do País; além de taxas ascendentes entre os idosos com 60 anos de idade e mais. Estes dados sustentam a necessidade de realização de novos estudos para avaliar a efetividade das campanhas de vacinação. Santos e colaboradores ${ }^{8}$ descrevem a atividade diurna de flebotomíneos potencialmente transmissores de leishmaniose tegumentar em áreas urbanas da cidade Maringá, Paraná. Os resultados, sobretudo relacionados a Nyssomyia whitmani, sugerem que a transmissão da leishmaniose tegumentar nas localidades estudadas possa ocorrer inclusive durante o dia. 0 estudo de Marreiro e colaboradores ${ }^{9}$ descreve a situação de encerramento dos casos de tuberculose após o processo de descentralização das ações de controle da doença na cidade Manaus, Amazonas. Os resultados mostraram taxa de cura abaixo da meta recomendada pela Organização Mundial de Saúde (OMS), assim como índice de abandono de tratamento superior ao preconizado pelo Programa Nacional de Controle da Tuberculose. Este estudo serviu também para identificar importantes falhas operacionais tais como subnotificações, inconsistências, além de incompletitudes e duplicidades de registros, achados que reforçam a importância da capacitação de recursos humanos e do monitoramento das ações no processo de descentralização. Gontijo e colaboradores ${ }^{10}$ realizaram inquérito sorológico com 63.673 neonatos em Minas Gerais com vistas a definir o risco de transmissão vertical da infecção pelo Trypanosoma cruzi, agente causador da doença de Chagas. 0 risco de transmissão vertical foi estimado em $0,2 \%$, sendo recomendado que a sorologia para diagnóstico de Doença de Chagas congênita seja incluída no Programa de Triagem Neonatal em áreas consideradas endêmicas. 0 estudo de Soares e colaboradores ${ }^{11}$ analisa as características epidemiológicas 
de 2.532 vítimas de acidentes e violências atendidas durante um mês em unidades de emergência dos Municípios de Cuiabá e Várzea Grande, Mato Grosso. Os resultados mostram que os adultos jovens e do sexo masculino foram as principais vítimas e a suspeição de uso de álcool prevaleceu nas violências. Os acidentes corresponderam a $90,3 \%$ dos registros, com destaque para as quedas $(54,8 \%)$ e os acidentes de transporte $(26,8 \%)$, dentre estes predominaram os de motocicleta $(45,9 \%)$. Os dados providos por este levantamento poderão ser utilizados para sensibilizar autoridades e gestores da área de saúde e de outras áreas para o desenvolvimento de políticas e programas de prevenção de acidentes e violências. Oliveira-Filho e Martinelli ${ }^{12}$ realizaram um levantamento dos casos notificados de malária no Estado do Pará, entre 1998 e 2006, com intuito de quantificar o risco de transmissão da doença, discriminar o número de casos por espécies de Plasmodium e destacar as áreas de maior incidência. Os resultados mostraram que o Estado do Pará apresentou redução significativa de casos notificados de malária no período analisado, com tendência de aumento do número de municípios com IPA baixa e média. Uma possível explicação para estes resultados seria a intensificação das ações governamentais de controle e prevenção à malária na Região.

Dois artigos originais avaliam aspectos relacionados à qualidade dos dados gerados através de sistemas de informação. Oliveira e colaboradores ${ }^{13}$ avaliam a completitude das fichas de investigação epidemiológica de febre tifóide notificados pelo Sinan na Bahia de 2003 a 2006. Verificaram que a maioria dos campos apresentou completitude regular (70\%-89\%) ou baixa (<70\%), sendo que apenas nas variáveis de identificação foi possível observar 100\% de completitude. Os resultados evidenciam a necessidade de investimentos na melhoria do preenchimento das fichas de investigação e esclarecimento dos profissionais de saúde sobre a importância destas informações para 0 controle da doença. Luquetti e Laguardia ${ }^{14}$ avaliaram a confiabilidade dos dados de atendimento odontológico registrados em três diferentes instrumentos. Os resultados revelam alta concordância entre os dados coletados por um instrumento padronizado ou desenvolvido localmente e os dados presentes nos relatórios gerados pelo Sistema de Gerenciamento de Unidade Ambulatorial Básica (Sigab).

Completando esta edição, os leitores terão acesso à republicação do artigo de Barcellos e colaboradores ${ }^{15} \mathrm{em}$ que são avaliados cenários de mudanças climáticas e ambientais para o Brasil. 0 trabalho visa também contribuir para a identificação de recursos que podem ser utilizados para desenvolver uma rede de diagnóstico, modelagem, análise e intervenção sobre as repercussões dessas mudanças sobre as condições de saúde da população brasileira. Dentre as doenças que potencialmente podem ser afetadas por estas mudanças, 0 artigo destaca as de veiculação hídrica, as respiratórias e as transmitidas por vetores, em especial a malária e a dengue.

Os estudos que compõem esta edição trazem, além de informações relevantes sobre temas importantes para a saúde pública, exemplos de diferentes estratégias para auxiliar a compreensão de problemas, e ainda métodos para avaliar a qualidade da informação gerada nos serviços de saúde.

\section{Guilherme Werneck}

Membro do Comitê Editorial

\section{Referências}

1. Barata RCB. O desafio das doenças emergentes e a revalorização da epidemiologia descritiva. Revista de Saúde Pública. 1997; 31(5):531-537.

2. MacMahon B, Pugh TFH. Epidemiology: principles and methods. Boston: Little Brown \& Co.; 1970.

3. Lilienfeld AM, Lilienfeld DE. Foundations of epidemiology. 2nd ed. New York: Oxford University Press; 1980.

4. Krieger N. Epidemiology and social sciences: towards a critical reengagement in the 21st century. Epidemiologic Reviews 2000; 22(1):155-163.

5. Kleinbaum DG, Kupper LL, Morgenstern H. Epidemiologic Research Principles and Quantitative Methods. Belmont: Lifetime Learning Publications; 1982. 
6. Schoenbach VJ, Rosamond WD. Understanding the Fundamentals of Epidemiology: an evolving text. Chapel Hill: North Carolina; 2000. [acessado em 27 Maio 2009]. Disponível em http://www.epidemiolog.net/evolving/FundamentalsOfEpi demiology.pdf

7. Campagna AS, Dourado I, Duarte EC, Daunfenbach LZ. Mortalidade por causas relacionadas à influenza em idosos no Brasil, 1992 a 2005. Epidemiologia e Serviços de Saúde 2009; 18(3):209-218.

8. Santos DR, Santos AR, Santos ES, Oliveira 0, Poiani LP, Silva AM. Observações sobre a atividade diurna de Nyssomyia whitmani (Diptera: Psychodidae) na área urbana de Maringá, Paraná, Brasil. Epidemiologia e Serviços de Saúde 2009; 18(3): 227-236.

9. Marreiro LS, Cruz MA, Oliveira MNF, Garrido MS. Tuberculose em Manaus, Estado do Amazonas: resultado de tratamento após a descentralização. Epidemiologia e Serviços de Saúde 2009; 18(3):237-242.

10. Gontijo ED, Andrade GMQ, Santos SE, Galvão LMC, Moreira EF, Pinto FS, Dias JCP, Januário JN. Triagem neonatal da infecção pelo Trypanosoma cruzi em Minas Gerais, Brasil: transmissão congênita e mapeamento das áreas endêmicas. Epidemiologia e Serviços de Saúde 2009; 18(3):243-254.

11. Soares BAC, Scatena JH, Galvão ND. Acidentes e violências na Grande Cuiabá: o que retrata a demanda dos serviços de emergência. Epidemiologia e Serviços de Saúde 2009; 18(3):265-276.

12. Oliveira-Filho AB, Martinelli JM. Casos notificados de malária no Estado do Pará, Amazônia Brasileira, de 1998 a 2006. Epidemiologia e Serviços de Saúde 2009; 18(3):277-284.

13. Oliveira MEP, Soares MRAL, Costa MCN, Mota ELA. Avaliação da completitude dos registros de febre tifóide notificados no Sinan pela Bahia. Epidemiologia e Serviços de Saúde 2009; 18(3):219-226.

14. Luquetti LB, Laguardia J. Confiabilidade dos dados de atendimento odontológico do Sistema de Gerenciamento de Unidade Ambulatorial Básica (Sigab) em Unidade Básica de Saúde do Município do Rio de Janeiro. Epidemiologia e Serviços de Saúde 2009; 18(3):255-264.

15. Barcellos C, Monteiro AMV, Corvalán C, Gurgel HC, Carvalho MS, Artaxo P, Hacon S, Ragoni V. Mudanças climáticas e ambientais e as doenças infecciosas: cenários e incertezas para o Brasil. Epidemiologia e Serviços de Saúde 2009; 18(3): 285-304. 